\title{
Concentraciones totales de Fe, Mn, Al y P en Alfisoles, Oxisoles y Ultisoles en Misiones, Argentina
}

\author{
Prause ${ }^{1}$, Juan; Fernández López, Carolina; Dalurzo, Humberto C. \\ ${ }^{1}$ Universidad Nacional del Nordeste. Facultad de Ciencias Agrarias. Departamento de Suelo y Agua. \\ Cátedra de Edafología. Sargento Cabral 2131 (3.400) Corrientes, Argentina
}

\begin{abstract}
Resumen
Uno de los principales problemas en la producción agrícola en la Provincia de Misiones (Argentina) es la baja disponibilidad de fósforo $(\mathrm{P})$ en Alfisoles, Oxisoles y Ultisoles, aunque se detectan altas concentraciones de $\mathrm{P}$ total. El objetivo del trabajo fue determinar la distribución de las concentraciones totales de Fe, Mn, Al y P en Alfisoles, Oxisoles y Ultisoles. Kandiudalfes Ródico y Mólico, Eutrudox Ródico, Hapludox Húmico, Kandihumultes Típicos. Se determinaron pH en agua relación 1:2,5; carbono orgánico (CO) por Walkley-Black; textura por Bouyouccos; Fósforo total (Pt) por digestión con $\mathrm{H}_{2} \mathrm{SO}_{4}-\mathrm{H}_{2} \mathrm{O}_{2}-\mathrm{HF}$. El fraccionamiento de $\mathrm{Fe}, \mathrm{Mn}$, y Al se realizó siguiendo el procedimiento propuesto por Shuman y modificado por McDaniel y Buol adaptado para el fraccionamiento de elementos. Las concentraciones de $\mathrm{Fe}, \mathrm{Mn}$ y Al fueron determinadas por espectrofotometría de absorción atómica. Se concluye que: i) las formas amorfas o pobremente cristalinas de Fe, fueron las formas más importantes hallada de este elemento; ii) las principales formas de Mn halladas fueron los óxidos cristalinos de Mn o formas coprecipitadas, encontrándose que las concentraciones totales de Mn disminuyeron en profundidad y están estrechamente relacionadas a los contenidos de arcillas del suelo; iii) para Al se detectaron las formas amorfas y las cristalinas de óxido de hierro o formas coprecipitadas; iv) en los tres órdenes de suelos la tercera fracción en orden de importancia fue el Al asociadas a la materia orgánica; v) la menor concentración de fósforo fue hallada en los Alfisoles y la mayor en los Ultisoles y su disponibilidad es afectada principalmente por la presencia de diferentes formas de Fe, Mn, y Al.
\end{abstract}

Palabras clave: perfiles de suelo, concentraciones, fósforo, micronutrientes.

\begin{abstract}
Summary
One of the main problems of the agricultural production in the Province of Misiones (Argentina) is the low availability of phosphorus (P) in Alfisols, Oxisols and Ultisols, although high concentrations of total $\mathrm{P}$ are detected. The objective of this work was to determine the distribution of total concentrations of Fe, Mn, Al and P in Alfisols, Oxisols and Ultisols. Analyzed soils were Rodic and Molic Kandiudalf, Molic Eutrudox, Humic Hapludox andTypic Kandihumult. pH in water was determined (1:2.5 ratio), as well as organic carbon (OC) by Walkley-Black, Bouyouccos texture, and total phosphorus (TP) by digestion with $\mathrm{H}_{2} \mathrm{SO}_{4}-\mathrm{H}_{2} \mathrm{O}_{2}$ - HF. The fractionation of $\mathrm{Fe}, \mathrm{Mn}$ and $\mathrm{Al}$ was performed following the procedure proposed by Shuman and modified by McDaniel and Buol, adapted for the fractionation of elements. Concentrations of $\mathrm{Fe}, \mathrm{Mn}$ and $\mathrm{Al}$ were determined by atomic absorption spectrophotometry. We concluded that: i) the amorphous or poorly crystalline forms of Fe were the most important forms of this element, ii) the main forms of Mn were crystalline Mn oxides or coprecipitated forms, finding that the total $\mathrm{Mn}$ concentration decreased in depth and was closely related to soil clay content, iii) the amorphous forms of $\mathrm{Al}$ were detected, and also the crystalline ferric oxide or coprecipitated forms; iv) in the three soil orders, Al associated with organic matter was the third fraction in order of importance; v) the lowest concentration of phosphorus was found in Alfisols and the highest in Ultisols, and its availability was mainly affected by the presence of different forms of $\mathrm{Fe}, \mathrm{Mn}$, and Al.
\end{abstract}

Key words: soil profiles, concentration, phosphorus, micronutrients.

\section{INTRODUCCIÓN}

La Provincia de Misiones se encuentra ubicada en una región con clima subtropical sin estación seca marcada, donde predominan, desde el punto de vista taxonómico los Alfisoles, Oxisoles y Ultisoles. Los suelos rojos están muy extendidos en los trópicos y la presencia de los óxidos de hierro en estos suelos, tie- nen su mayor efecto sobre la estabilidad y fertilidad de los mismos. Los óxidos de hierro son también usados como indicadores de los procesos de formación y del medio ambiente pedogenético del suelo, aunque la influencia de las lluvias y del material parental sobre las propiedades de los óxidos de hierro son pobremente comprendidas (Trakoonyingcharoen et al., 
2006). La aptitud para su uso está condicionada por factores tales como baja fertilidad, alta acidez y susceptibilidad a la erosión hïdrica (Ligier et al., 1990). En muchos suelos ácidos los óxidos, hidróxidos y oxihidróxidos de $\mathrm{Fe}$, Mn y Al constituyen un grupo de coloides que son capaces de adsorber amplias cantidades de metales traza y determinan las concentraciones de los mismos en la solución. Este grupo de coloides tiene una importante influencia en la sorción y disponibilidad de fosfatos (Yuan y Lavkulich, 1994). La sorción de metales traza y de fosfatos en varios suelos está influenciada por la presencia de diferentes formas de $\mathrm{Fe}, \mathrm{Mn}$ y Al en cantidades variables y por el $\mathrm{pH}$, materia orgánica, y otras propiedades del suelo (Wang et al., 1991; Backes et al., 1995; Zhang et al., 1997).

El contenido total de $\mathrm{Fe}$ en el suelo varía desde menos de $0,1 \%$ en algunos suelos arenosos lavados, hasta más del $30 \%$ en horizontes enriquecidos en $\mathrm{Fe}$ y plintitas. Es un componente importante tanto de silicatos primarios como de minerales de arcilla. Sus compuestos principales son óxidos e hidróxidos, frecuentemente hidratados y por lo común finamente divididos en los suelos donde tienen un papel decisivo en la coloración de los mismos (Charlet y Sposito, 1987). El hecho que el Fe esté presente como elemento nativo y con dos estados de valencia, hacen que el metal y sus compuestos sean indicadores sensibles de las condiciones de oxidación en ambientes naturales (Mortvedt et al.,. 1983). La fracción coloidal de los óxidos de $\mathrm{Fe}$ en suelos caoliníticos se deposita en la superficie de estas arcillas y contribuye a su agregación (Fassbender y Bornemisza, 1987).

El contenido normal de $\mathrm{Mn}$ en el suelo está entre 300 y $500 \mu \mathrm{g} \mathrm{g}^{-1}$ pero puede variar entre límites mucho más amplios. Se encuentra como $\mathrm{Mn}^{2+}$ en la solución, sobre el complejo de cambio y en forma de diferentes óxidos en los que pueden presentarse también como $\mathrm{Mn}^{3+} \mathrm{y} \mathrm{Mn}^{4+}$, de acuerdo a lo expresado por Shuman y Anderson en 1978 (citado por Wild, 1992). Es fácil que aparezcan síntomas de toxicidad en los suelos ácidos como consecuencia de la reducción de los óxidos de Mn. La estabilidad de varias formas de $\mathrm{Fe}$ y $\mathrm{Mn}$ en los suelos es dependiente del pH y del Eh de los mismos (Zhang et al., 1997). Entre las propiedades que inciden sobre los niveles de Mn se encuentran el $\mathrm{pH}$, la textura y el contenido de materia orgánica (Fassbender y Bornemisza, 1987).

El factor más perjudicial para las plantas que se desarrollan en suelos ácidos es el contenido de $\mathrm{Al}$ en concentraciones tóxicas, particularmente cuando el $\mathrm{pH}$ es inferior a 5,5. En estas condiciones el Al limita la descomposición de la materia orgánica (Alexander, 1980). El pH en el que los niveles de Al alcanzan valores perjudiciales depende tanto de la planta como de una serie de factores del suelo como la mineral- ogía de las arcillas, el contenido de materia orgánica, la presencia de otros cationes y aniones y la salinidad total del suelo (Fassbender y Bornemisza, 1987). Arnseth and Turner (1988) indicaron que la distribución vertical y la variación de los distintos compuestos de $\mathrm{Fe}, \mathrm{Mn}$ y Al eran indicadores sensitivos de los procesos pedogenéticos.

Los suelos contienen entre 100 y $3000 \mathrm{mg} \mathrm{P} \mathrm{kg}$ de suelo, la mayoría del cual está presente como ortofosfato. Su baja concentración y solubilidad lo hacen uno de los nutrientes más limitantes (Fernández López, et al., 2000). En la mayoría de los ecosistemas naturales, el ciclo del $\mathrm{P}$ es esencialmente cerrado con mínimas pérdidas o ganancias (Wild, 1992). En suelos no fertilizados, se presentan los valores más altos cuando el material parental está formado por rocas eruptivas de reacción básica, y en suelos con elevado contenido de materia orgánica. Se produce una reducción del contenido de fosfatos a medida que las rocas se meteorizan, ya que la apatita, principal mineral fosfatado de estas rocas, se disuelve en el medio ácido de las aguas de lixiviación antes de la formación de óxidos de hierro y aluminio o de minerales de naturaleza arcillosa que podrían adsorber los iones fosfatos (Wild, 1992). En el perfil del suelo, la mayor parte de los fosfatos está generalmente próxima a la superficie, atribuyéndose en los suelos cultivados, en parte por las prácticas de fertilizaciones fosfatadas, y en los suelos no cultivados como consecuencia que los fosfatos son reciclados a través de la vegetación, depositándose tras la caída de las hojas u otros residuos (Schlesinger, 2000).

El valor de $\mathrm{P}$ total en los suelos de áreas tropicales parece estar ligado al contenido de materia orgánica y a su evolución pedológica. En un estudio sobre suelos de América Central, Fassbender (1966) reportó que en los suelos más ácidos, predominaban los fosfatos orgánicos, y dentro de los inorgánicos los de aluminio, hierro. El contenido de $\mathrm{P}$ total era mayor, llegando en promedio a $1,241 \mathrm{mg} \mathrm{g}^{-1}$, encontrándose mayores concentraciones de $\mathrm{P}$ total cuando la textura era más fina. La hipótesis del trabajo fue que las concentraciones de Fe, Mn, Al y P en Alfisoles, Oxisoles y Ultisoles varían con la profundidad del perfil del suelo afectando la disponibilidad de fósforo en diversa magnitud. El objetivo del trabajo fue determinar la distribución de las concentraciones totales de Fe, Mn, Al y $\mathrm{P}$ en perfiles de Alfisoles, Oxisoles y Ultisoles.

\section{MATERIALES Y MÉTODOS}

En la Provincia de Misiones (Argentina) de los Departamentos de Leandro N. Alem y Candelaria, a $27^{\circ} 20^{\prime}$ y $28^{\circ} 10^{\prime}$ de latitud sur y $55^{\circ} 10^{\prime}$ y $56^{\circ} 00^{\prime}$ de longitud oeste se tomaron 60 muestras de suelos representitvos de 4 perfiles de cada orden clasificados como Alfisoles, Oxisoles y Ultisoles bajo diferentes 
condiciones de campo. Los suelos fueron muestreados hasta alcanzar el horizonte $\mathrm{C}$ (Tabla 1). Las propiedades morfológicas de los perfiles de suelo fueron descriptas y clasificadas de acuerdo con el Soil Taxonomy (Soil Survey Staff, 2006). Los Alfisoles incluyeron: Kandiudalf Ródico y Kandiudalf Mólico. Los Oxisoles muestreados fueron: Eutrudox Mólico y Hapludox Húmico. Los Ultisoles muestreados fueron: Kandiumultes Típicos. Las muestras de suelo fueron secadas al aire, molidas y pasadas por tamiz de $2 \mathrm{~mm}$ y sometidas a los siguientes análisis: $\mathrm{pH}$ en extracto acuoso (1:2.5); carbono orgánico (CO) por WalkleyBlack (Nelson y Sommers, 1996); textura por el método de Bouyoucos (Forsythe, 1975); fósforo total (Pt) determinado por digestión con $\mathrm{H}_{2} \mathrm{SO}_{4}-\mathrm{H}_{2} \mathrm{O}_{2}$ - HF (Bartels, et al., 1996). Los datos del fraccionamiento de Fe, Mn, y Al fueron analizados siguiendo el procedimiento propuesto por Shuman (1985) y modificado por McDaniel y Buol (1991), adaptado para el fraccionamiento de elementos (Zhang, et al., 1997). Las concentraciones de Fe, Mn and Al fueron determinadas por espectrofotometría de emisión. El fraccionamiento y la actividad iónica fueron analizados usando el INFOSTAT 2009 (Di Rienzo et al., 2009).

\section{RESULTADOS Y DISCUSIÓN}

Las propiedades del suelo, el fósforo total $(\mathrm{Pt}), \mathrm{y}$ la suma de las fracciones de las diferentes formas de Fe, Mn y Al de cada uno de los horizontes de los órdenes de suelo seleccionados para este estudio, son presentados en la Tabla 1. El material originario de estos suelos fue roca basáltica, pero debido a la intensa meteorización como consecuencia de las altas temperaturas y precipitaciones pluviales, el $\mathrm{pH}$ de los

Tabla 1: Propiedades físicas, químicas y datos de fraccionamiento de Fe, Mn, Al y P en Alfisoles, Oxisoles y Ultisoles de Misiones, Argentina.

\begin{tabular}{|c|c|c|c|c|c|c|c|c|c|c|}
\hline Horizonte & $\begin{array}{l}\text { Prof. } \\
(\mathrm{cm})\end{array}$ & $\mathrm{pH}$ & $\begin{array}{l}\mathrm{CO} \\
(\%) \\
\end{array}$ & $\begin{array}{l}\text { Arena } \\
(\end{array}$ & $\begin{array}{r}\text { Limo } \\
\% \\
\end{array}$ & $\begin{array}{c}\text { Arcilla } \\
\text { ) }\end{array}$ & $\begin{array}{l}\mathrm{P} \\
(\end{array}$ & \multicolumn{2}{|c|}{$\mathrm{mg} \mathrm{kg}^{-1}$} & $\begin{array}{r}\mathrm{Al} \\
) \\
\end{array}$ \\
\hline \multicolumn{11}{|c|}{ Alfisol } \\
\hline A & $0-23$ & $\begin{array}{c}4,5 * \\
(0,21) * *\end{array}$ & $\begin{array}{r}1,67 \\
(0,31) \\
\end{array}$ & $\begin{array}{l}22,00 \\
(4,06) \\
\end{array}$ & $\begin{array}{l}15,20 \\
2,83) \\
\end{array}$ & $\begin{array}{r}62,80 \\
(6,63) \\
\end{array}$ & $\begin{array}{c}484 \\
(19,7) \\
\end{array}$ & $\begin{array}{c}913 \\
(140,7) \\
\end{array}$ & $\begin{array}{c}1141 \\
(148,7) \\
\end{array}$ & $\begin{array}{c}2465 \\
(368,7) \\
\end{array}$ \\
\hline $\mathrm{BA}$ & $23-48$ & $\begin{array}{c}4,75 \\
(0,16) \\
\end{array}$ & $\begin{array}{c}0,94 \\
(0,11)\end{array}$ & $\begin{array}{l}14,50 \\
(2,93)\end{array}$ & $\begin{array}{l}13,20 \\
(0,83)\end{array}$ & $\begin{array}{l}72,30 \\
(2,26)\end{array}$ & $\begin{array}{c}459 \\
(23,3)\end{array}$ & $\begin{array}{c}1522 \\
(779,2)\end{array}$ & $\begin{array}{c}842 \\
(102,8)\end{array}$ & $\begin{array}{c}2672 \\
(868,9)\end{array}$ \\
\hline $\mathrm{Bt}$ & $48-86$ & $\begin{array}{l}4,94 \\
(0,19)\end{array}$ & $\begin{array}{c}0,75 \\
(0,02) \\
\end{array}$ & $\begin{array}{l}16,10 \\
(2,96) \\
\end{array}$ & $\begin{array}{l}10,40 \\
(2,34) \\
\end{array}$ & $\begin{array}{l}73,50 \\
(1,03) \\
\end{array}$ & $\begin{array}{c}414 \\
(49,8) \\
\end{array}$ & $\begin{array}{c}983 \\
(442,4) \\
\end{array}$ & $\begin{array}{c}762 \\
(360,3) \\
\end{array}$ & $\begin{array}{c}2448 \\
(296,6) \\
\end{array}$ \\
\hline $\mathrm{BC}$ & $\begin{array}{l}86- \\
104 \\
\end{array}$ & $\begin{array}{c}4,76 \\
(0,26) \\
\end{array}$ & $\begin{array}{c}0,67 \\
(0,05) \\
\end{array}$ & $\begin{array}{l}14,50 \\
(0,51) \\
\end{array}$ & $\begin{array}{l}10,80 \\
(1,77) \\
\end{array}$ & $\begin{array}{l}74,80 \\
(1,35) \\
\end{array}$ & $\begin{array}{c}409 \\
(59,7) \\
\end{array}$ & $\begin{array}{c}734 \\
(94,3) \\
\end{array}$ & $\begin{array}{c}606 \\
(319,4) \\
\end{array}$ & $\begin{array}{c}1962 \\
(254,5) \\
\end{array}$ \\
\hline $\mathrm{CB}$ & $\begin{array}{c}104- \\
140 \\
\end{array}$ & $\begin{array}{c}4,91 \\
(0,17) \\
\end{array}$ & $\begin{array}{c}0,52 \\
(0,06) \\
\end{array}$ & $\begin{array}{l}13,80 \\
(4,29) \\
\end{array}$ & $\begin{array}{l}12,00 \\
(3,85) \\
\end{array}$ & $\begin{array}{l}74,20 \\
(1,34) \\
\end{array}$ & $\begin{array}{c}389 \\
(52,0) \\
\end{array}$ & $\begin{array}{c}1093 \\
(120,1) \\
\end{array}$ & $\begin{array}{c}595 \\
(311,7) \\
\end{array}$ & $\begin{array}{c}2223 \\
(215,5) \\
\end{array}$ \\
\hline \multicolumn{11}{|c|}{ Oxisol } \\
\hline A & $0-19$ & $\begin{array}{c}4,79 \\
(0,40) \\
\end{array}$ & $\begin{array}{c}1,67 \\
(0,20) \\
\end{array}$ & $\begin{array}{l}12,00 \\
(1,58) \\
\end{array}$ & $\begin{array}{l}15,90 \\
(3,23) \\
\end{array}$ & $\begin{array}{r}72,10 \\
(4,09) \\
\end{array}$ & $\begin{array}{c}645 \\
(34,0) \\
\end{array}$ & $\begin{array}{c}686 \\
(180,8) \\
\end{array}$ & $\begin{array}{c}458 \\
(54,1) \\
\end{array}$ & $\begin{array}{c}1599 \\
(982,4) \\
\end{array}$ \\
\hline BA & $19-31$ & $\begin{array}{c}4,63 \\
(0,19) \\
\end{array}$ & $\begin{array}{c}1,30 \\
(0,47) \\
\end{array}$ & $\begin{array}{l}11,40 \\
(1,43) \\
\end{array}$ & $\begin{array}{l}13,50 \\
(1,15) \\
\end{array}$ & $\begin{array}{l}75,10 \\
(1,23) \\
\end{array}$ & $\begin{array}{c}614 \\
(56,8) \\
\end{array}$ & $\begin{array}{c}618 \\
(260,4) \\
\end{array}$ & $\begin{array}{c}417 \\
(57,3) \\
\end{array}$ & $\begin{array}{c}1442 \\
(981,9) \\
\end{array}$ \\
\hline B & $31-51$ & $\begin{array}{c}4,85 \\
(0,10)\end{array}$ & $\begin{array}{c}1,11 \\
(0,30)\end{array}$ & $\begin{array}{c}9,50 \\
(2,68)\end{array}$ & $\begin{array}{l}13,20 \\
(2,02)\end{array}$ & $\begin{array}{l}77,30 \\
(0,66)\end{array}$ & $\begin{array}{c}589 \\
(60,6)\end{array}$ & $\begin{array}{c}791 \\
(312,1)\end{array}$ & $\begin{array}{c}311 \\
(109,4)\end{array}$ & $\begin{array}{c}2017 \\
(1186,0)\end{array}$ \\
\hline $\mathrm{BC}$ & $\begin{array}{l}51- \\
103 \\
\end{array}$ & $\begin{array}{c}4,85 \\
(0,10) \\
\end{array}$ & $\begin{array}{c}0,93 \\
(0,14) \\
\end{array}$ & $\begin{array}{c}8,70 \\
(4,65) \\
\end{array}$ & $\begin{array}{l}11,70 \\
(1,23) \\
\end{array}$ & $\begin{array}{l}79,60 \\
(3,43) \\
\end{array}$ & $\begin{array}{c}501 \\
(56,4) \\
\end{array}$ & $\begin{array}{c}1030 \\
(902,4)\end{array}$ & $\begin{array}{c}95 \\
(53,3) \\
\end{array}$ & $\begin{array}{c}2034 \\
(1327,0) \\
\end{array}$ \\
\hline $\mathrm{CB}$ & $\begin{array}{l}103- \\
138 \\
\end{array}$ & $\begin{array}{c}4,70 \\
(0,14) \\
\end{array}$ & $\begin{array}{c}0,80 \\
(0,15) \\
\end{array}$ & $\begin{array}{c}9,20 \\
(2,25) \\
\end{array}$ & $\begin{array}{l}10,50 \\
(1,08) \\
\end{array}$ & $\begin{array}{l}80,30 \\
(2,22) \\
\end{array}$ & $\begin{array}{c}468 \\
(30,1) \\
\end{array}$ & $\begin{array}{c}682 \\
(247,0) \\
\end{array}$ & $\begin{array}{c}116 \\
(80,6) \\
\end{array}$ & $\begin{array}{c}1737 \\
(1087,0) \\
\end{array}$ \\
\hline \multicolumn{11}{|c|}{ Ultisol } \\
\hline A & $0-16$ & $\begin{array}{c}4,60 \\
(0,23)\end{array}$ & $\begin{array}{c}2,45 \\
(0,53)\end{array}$ & $\begin{array}{l}13,40 \\
(0,13)\end{array}$ & $\begin{array}{l}22,10 \\
(3,58)\end{array}$ & $\begin{array}{l}64,60 \\
(3,70)\end{array}$ & $\begin{array}{c}796 \\
(173,0)\end{array}$ & $\begin{array}{c}1240 \\
(188,0)\end{array}$ & $\begin{array}{c}737 \\
(396,4) \\
\end{array}$ & $\begin{array}{c}3386 \\
(272,3) \\
\end{array}$ \\
\hline BA & $16-41$ & $\begin{array}{c}4,61 \\
(0,14) \\
\end{array}$ & $\begin{array}{c}1,65 \\
(0,15) \\
\end{array}$ & $\begin{array}{l}16,50 \\
(2,23) \\
\end{array}$ & $\begin{array}{l}13,80 \\
(6,75) \\
\end{array}$ & $\begin{array}{r}69,80 \\
(5,57) \\
\end{array}$ & $\begin{array}{c}664 \\
(38,1) \\
\end{array}$ & $\begin{array}{c}1072 \\
(133,6) \\
\end{array}$ & $\begin{array}{c}371 \\
(130)\end{array}$ & $\begin{array}{c}3046 \\
(332,9) \\
\end{array}$ \\
\hline $\mathrm{Bt}$ & $41-61$ & $\begin{array}{c}4,55 \\
(0,06)\end{array}$ & $\begin{array}{c}1,30 \\
(0,25)\end{array}$ & $\begin{array}{l}14,50 \\
(0,63)\end{array}$ & $\begin{array}{l}14,20 \\
(5,28)\end{array}$ & $\begin{array}{l}71,30 \\
(4,88) \\
\end{array}$ & $\begin{array}{c}607 \\
(36,4)\end{array}$ & $\begin{array}{c}1089 \\
(119,2)\end{array}$ & $\begin{array}{c}251 \\
(83,5)\end{array}$ & $\begin{array}{c}3255 \\
(228,2)\end{array}$ \\
\hline $\mathrm{BC}$ & $61-88$ & $\begin{array}{c}4,57 \\
(0,08) \\
\end{array}$ & $\begin{array}{c}0,99 \\
(0,10) \\
\end{array}$ & $\begin{array}{l}10,00 \\
(2,20) \\
\end{array}$ & $\begin{array}{l}15,80 \\
(3,13) \\
\end{array}$ & $\begin{array}{r}74,30 \\
(5,32) \\
\end{array}$ & $\begin{array}{c}572 \\
(28,1) \\
\end{array}$ & $\begin{array}{c}574 \\
(70,6) \\
\end{array}$ & $\begin{array}{c}197 \\
(43,4) \\
\end{array}$ & $\begin{array}{c}2534 \\
(348,4) \\
\end{array}$ \\
\hline $\mathrm{CB}$ & $\begin{array}{l}88- \\
120\end{array}$ & $\begin{array}{c}4,71 \\
(0,10)\end{array}$ & $\begin{array}{c}0,79 \\
(0,07)\end{array}$ & $\begin{array}{c}9,30 \\
(2,34)\end{array}$ & $\begin{array}{l}14,30 \\
(1,98)\end{array}$ & $\begin{array}{l}76,50 \\
(2,72)\end{array}$ & $\begin{array}{c}568 \\
(44,7)\end{array}$ & $\begin{array}{c}820 \\
(412,1)\end{array}$ & $\begin{array}{c}176 \\
(93,0)\end{array}$ & $\begin{array}{c}3029 \\
(194,6)\end{array}$ \\
\hline
\end{tabular}

(*) Valores promedios. (**) Desvíos estándart. 
suelos variaron entre 4,57 y 5,00 . Los perfiles muestreados fueron extremadamente arcillosos, registrándose en el horizonte A de los tres órdenes de suelos estudiados, porcentajes de arcilla que variaron entre $63 \%$ a $72 \%$. A partir de los $100 \mathrm{~cm}$ de profundidad se registraron los máximos valores de arcilla en Oxisoles $(80,30 \%)$ para los Ultisoles $(76,50 \%)$ y en los Alfisoles $(74,80 \%)$. El contenido de carbono orgánico (CO) de los horizontes A de los suelos estudiados varió entre $1,67 \%$ a $2,45 \%$.

Los resultados de la distribución de las concentraciones totales de, $\mathrm{Fe}, \mathrm{Mn}, \mathrm{y} \mathrm{Al}$ en los horizontes de los tres órdenes de suelos se muestran en la Figura 1. La distribución de las concentraciones de Fe, Mn, y
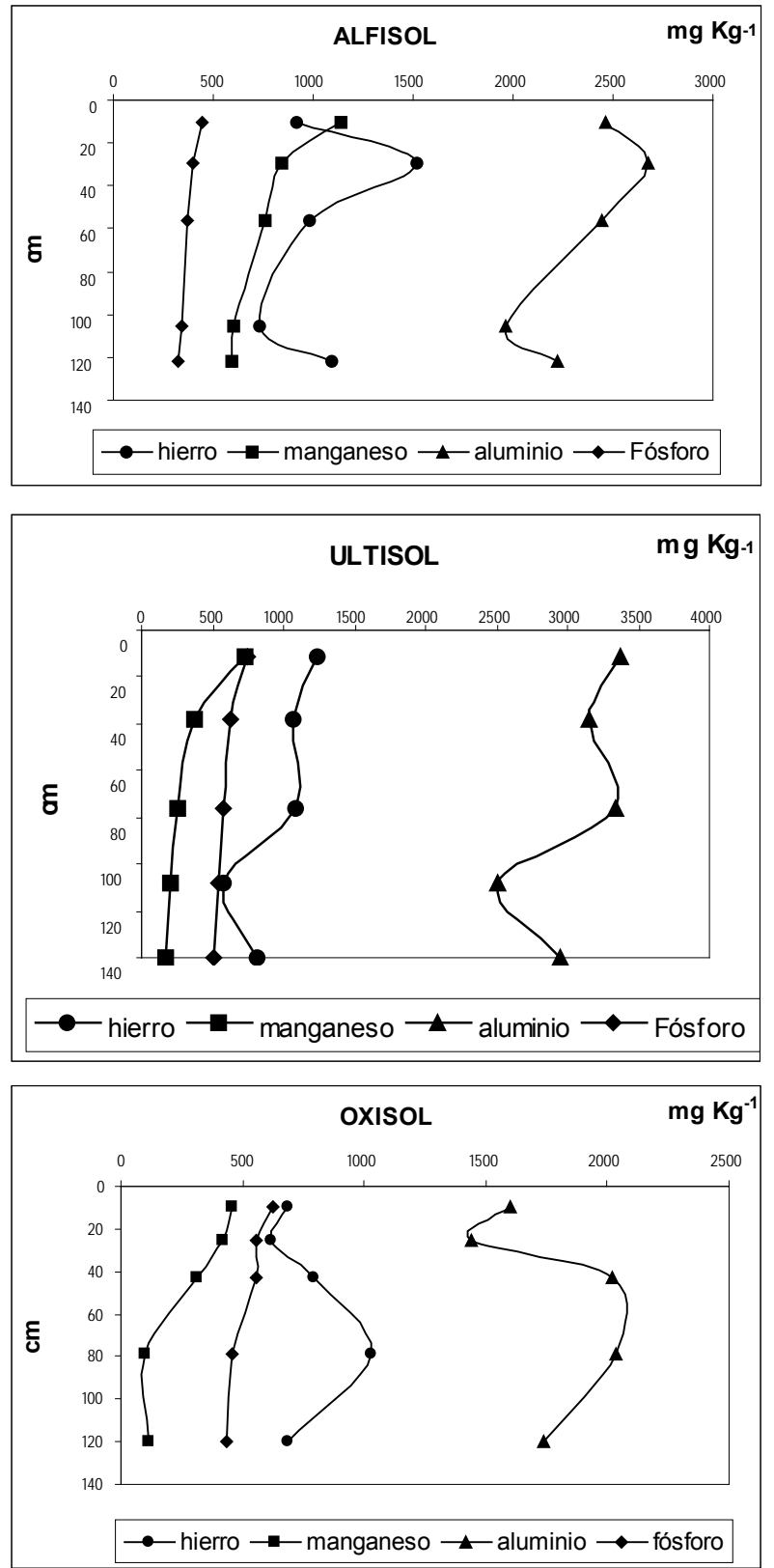

Figura 1: Distribución por profundidades de las concentraciones de $\mathrm{Fe}, \mathrm{Mn}, \mathrm{Al}$ en los perfiles de los suelos estudiados.
Al por profundidades en los perfiles estudiados, representan un reflejo de los procesos pedogenéticos (Yuan and Lavkulich, 1994). En el horizonte superficial (Ap), los patrones de distribución de estos elementos pueden ser diferentes que en los horizontes subsuperficiales, como consecuencia de la aplicación de diferentes prácticas culturales, a las que fueron sometidos algunos de ellos.

Alfisoles: El Fe se presentó en todo el perfil predominantemente asociado a las formas amorfas y en menor concentración como óxido de Fe. La cantidad de Fe hallada, asociada a los óxidos de Mn, fue insignificante, en concordancia con lo reportado por Zhang, et al., (1997) quienes encontraron que la proporción relativa al $\mathrm{Fe}$ amorfo fue mucho mayor en los horizontes subsuperficiales de los Alfisoles. Hay una considerable similitud entre el Fe y Al con respecto a la distribución de sus formas en diferentes profundidades de los perfiles. La principal diferencia es que, si bien una cantidad importante de $\mathrm{Al}$ se encontró en forma amorfa, también se halló y en una proporción similar, asociado a los óxidos de Fe. La acumulación de Fe y $\mathrm{Al}$ fue evidente en el horizonte $\mathrm{B} t$ de los $\mathrm{Al}$ fisoles. Las formas amorfas de $\mathrm{Al}$ y el $\mathrm{Al}$ asociadas a los óxidos de $\mathrm{Fe}$, representaron la principal fuente de este elemento, en el horizonte $\mathrm{B} t$ de los Alfisoles, según lo encontrado por Zhang, et al., (1997). Con respecto al Mn, este elemento mostró una fuerte afinidad con el contenido de la materia orgánica en el horizonte superficial. Las formas de Mn asociadas a la materia orgánica y al $\mathrm{Mn}$ intercambiable, se hallaron bien representadas en $\operatorname{los} 10 \mathrm{~cm}$ de profundidad. El Mn intercambiable disminuyó en profundidad, coincidiendo con lo encontrado por McDaniel y Buol (1991). Las principales formas de Mn son los óxidos de Mn, los amorfos y las asociadas a los óxidos de Fe.

Ultisoles: El Fe se encontró en todo el perfil, predominantemente como formas amorfas y sólo en los horizontes superficiales (hasta $10 \mathrm{~s} 40 \mathrm{~cm}$ de profundidad) se hallaron óxidos de Fe y Mn. En produndidad estas dos formas de Fe fueron insignificantes, encontrándose sólo como Fe amorfo. Zhang, et al., (1997) hallaron la misma distribución del Fe amorfo, pero en perfiles de suelos con $\mathrm{pH}>6,5$ y lo atribuyeron a que pueden existir diferentes patrones de distribución de los elementos como consecuencia de diversas prácticas culturales, principalmente fertilizaciones y riegos. Los cambios en las proporciones relativas de las varias formas de Al siguieron un patrón similar al del Al estudiado en los Alfisoles, con predominancia de las formas de $\mathrm{Al}$ amorfos y asociados a los óxidos de Fe, en una marcada menor concentración y disminuyendo en forma paulatina, las formas de $\mathrm{Al}$ asociadas a la materia orgánica, a los óxidos de Mn y el intercambiable. 
La forma intercambiable de $\mathrm{Mn}$ fue predominante en el horizonte superficial de los Ultisoles, en coincidencia con lo reportado por McDaniel y Buol (1991). En orden de importancia continuaron, los óxidos de $\mathrm{Mn}$, los amorfos y la asociada a la materia orgánica y a los óxidos de Fe.

Oxisoles: El Fe amorfo fue la forma predominante en todo el perfil de suelo acompañado por óxidos de Fe y en muy pequeña proporción a los óxidos de Mn. La mayor concentración de Fe se encontró a los 79 $\mathrm{cm}$ de profundidad del perfil, siendo atribuído a un intenso proceso de ferralitización según lo reportado por Wild (1992). Con respecto al Al, la mayor concentración hallada correspondió a las formas amorfas y asociadas a los óxidos de Fe continuando con el mismo patrón de distribución hallado para los perfiles anteriores. Aunque también se hallaron, en menor proporción, las asociadas a la materia orgánica, las intercambiables y la asociada a los óxidos de Mn. En este orden de suelo también la forma intercambiable de Mn fue la que predominó en el horizonte superficial, siguiéndole en importancia los óxidos de $\mathrm{Mn}$, los amorfos y los asociados a los óxidos de $\mathrm{Fe}$ y a la materia orgánica. Las concentraciones de Mn disminuyeron notablemente a partir de $10 \mathrm{~s} 79 \mathrm{~cm}$ de produndidad, atribuído a la fuerte presencia de un horizonte arcilloso.

\section{CONCLUSIONES}

1.- El Fe amorfo como óxido de Fe, fue la forma más importante hallada de este elemento. En los tres órdenes de suelo su concentración disminuye con la profundidad.

2.- Las principales formas de Mn detectadas fueron las amorfas y los óxidos de Mn. La disminución de las concentraciones totales de Mn en profundidad, está estrechamente relacionada a los contenidos de arcillas registrados a partir de los $100 \mathrm{~cm}$ de profundidad en el perfil de suelo.

3.- El Al siguió el mismo patrón de formas halladas que para el Fe. En los tres órdenes de suelos se detectaron las formas asociadas del $\mathrm{Al}$ con la materia orgánica.

4.- La menor concentración de fósforo fue hallada en los Alfisoles y la mayor en los Ultisoles, y su disponibilidad es afectada principalmente por la presencia de diferentes formas de Fe, Mn, y Al.

\section{BIBLIOGRAFÍA}

Alexander, M. 1980. Introducción a la Microbiología del Suelo. AGT Editor, S.A. México. pp. 491.

Arnseth, R. W. and Turner, R. S. 1988. Sequential extraction of iron, manganese, aluminum, and silicon in soils from two contrasting watersheds. Soil Sci. Soc. Am. J. 52: 1801-1807.
Backes, C.A.; McLaren, R. G.; Rate, A. W.; and Swift, R. S. 1995. Kinetics of cadmium and cobalt desorption from iron and manganese oxides. Soil Sci. Soc. Am. J. 59: 778-785.

Bartels, J. M. (Managing Editor). 1996. Methods of Soil Analysis. Part 3. Chemical Methods. Soil Science Society of America, Inc. Madison, Wisconsin, USA. Pp. 1390.

Charlet, L and Sposito, G. 1987. Monovalent Ion Adsorption by an Oxisol. Soil Science Society of America, Vol. 51, 1155-1160.

Di Rienzo J.A., Casanoves F., Balzarini M.G., Gonzalez L., Tablada M., Robledo C.W. INFOSTAT versión 2009. Grupo InfoStat, FCA, Universidad Nacional de Córdoba, Argentina.

Fassbender, H.W. 1966. Descripción físico-química del sistema fertilizante fosfatado-suelo-planta. Turrialba. Vol. 16, No 3, 237-246.

Fassbender, H.W. y Bornemisza, E. 1987. Química de suelos, con énfasis en suelos de América Latina. Instituto Interamericano de Cooperación para la Agricultura. San José, costa Rica. pp. 420.

Fernández López, C.; Vazquez, S.; Dalurzo, H. y L.A. Morales. 2000. Índice de disponibilidad del fósforo proveniente del fertilizante en suelos de la provincia de Misiones, Argentina. Agricultura Técnica, Chile. Vol. 61: 229-234.

Forsythe, W. 1975. Física de Suelos. Instituto Interamericano de Ciencias Agrícolas. San José, Costa Rica. Pp. 212.

Ligier, H., Matteio, H., Polo, H. and Rosso, J. 1990. Atlas de Suelos de la República Argentina. Escala 1: 500.000 y 1:1.000.000. Tomo II. Secretaría de Agriculturea, Ganadería y Pesca de la Nación. Proyecto PNUD/ARG 85/019. INTA. Centro de Investigaciones de Recursos Naturales. Imprenta Paz. Buenos Aires, Argentina. 109-154 págs.

McDaniel, P. A. and Buol, S.W. 1991. Manganese Distributions in Acid soils of the North Carolina Piedmont. Soil Science Society of America Journal. Vol. 55:152-158.

Mortvedt, J. J.; Giordano, P. M.; Lindsay, W. L. 1983. Micronutrientes en Agricultura. AGT Editor, S. A. México. pp. 742.

Nelson, D.W. and Sommers, L.E. 1996. Total Carbon, Organic Carbon, and Organic Matter. In: D.L. Sparks (Ed.). METHODS OF Soil Analysis. Part 3. Chaemical Methods. Chapter 34. ASA, SSSA, CSSA, Madison WI, pp. 961-1010.

Shuman, L. M. 1985. Fraccionation Method for Soil Microelements. Soil Science Society of America Journal. Vol. 140. $\mathrm{N}^{\circ} 1,11-22$.

Soil Survey Staff. 2006. Soil Taxonomy: A basic system of soil clasification for making and interpreting soil surveys. USDA-SCS, Agric. Handbook. 436. U.S. Gov. Print. Office, Washington, DC, pp.75. 
Schlesinger, W. H. 2000. Biogeoquímica.Un análisis del cambio global. Editorial Ariel, S.A. Barcelona, España. $577 \mathrm{pp}$.

Trakoonyingcharoen, P.; Kheoruenromne, I.; Suddhiprakarn, A. and Gilkes, R. J. 2006. Properties of iron oxides in red Oxisols and red Ultisols as affected by rainfall and soil parental material. Australian Journal of Soil Research 44: 63-70.

Yuan, G. and Lavkulich, L. M. 1994. Phosphate Sorption in Relation to Extractable Iron and aluminum in Spodosols. Soil Science Society of America Journal, Vol. 58, 343-346.
Wang, H. D.; Harris, W. G.; and Yuan, T. L. 1991. Relation between phosphorus and iron in Florida phosphatic soils. Soil sci. Soc. Am. J. 55:554-560.

Wild, A. 1992. Condiciones del Suelo y Desarrollo de las Plantas según Russell. Ediciones Mundi-Prensa. Madrid, España. pp 1045.

Zhang, M.; Alva, A. K.; Li, Y. C.; Calvert, D. V. 1997. Fractionation of Iron, Manganese, Aluminium, and Phosphorus in Selected sandy soils under Citrus Production. . Soil Science Society of America Journal, Vol.61 No 3, $794-801$. 